\title{
Adhesion Between Surfaces Separated by Molecularly Thin Perfluoropolyether Films
}

\author{
R. Brunner · G. W. Tyndall · R. J. Waltman • \\ F. E. Talke
}

Received: 30 July 2009/Accepted: 29 December 2009/Published online: 26 January 2010

(C) The Author(s) 2010

\begin{abstract}
The relationship between the adhesion of surfaces separated by a molecularly thin liquid film and the surface energy of the film was investigated. AFM-based force-distance curves were measured on a series of carbon surfaces coated with hydroxyl-terminated perfluoropolyether (PFPE) films. The surface energy of the PFPE films was varied by altering either the total film thickness or the bonding ratio of the film by changing the concentration of the PFPE film in the solution and/or the pull-rate during dip-coating. A linear relationship between adhesion force and surface energy was observed. Adhesion was found to vanish at non-zero values of surface energy. The experimental results indicate that the adhesive force between macroscopic bodies separated by molecularly thin liquid films is linearly proportional to the excess surface energy of the film.
\end{abstract}

Keywords Adhesion force - Surface energy · Disjoining pressure - Atomic force microscopy · Perfluoropolyether lubricant · Carbon overcoat

R. Brunner $(\bowtie) \cdot$ F. E. Talke

Center for Magnetic Recording Research, 9500 Gilman Drive

M/C 0401, La Jolla, CA 92093-0401, USA

e-mail: rbrunner@ucsd.edu

G. W. Tyndall

Western Digital Corporation, 5863 Rue Ferrari, San Jose, CA 95138, USA

R. J. Waltman

Hitachi Global Storage Technologies, 5600 Cottle Road,

San Jose, CA 95193, USA

\section{Introduction}

The adhesion between two solid surfaces in the presence of a molecularly thin liquid film is of extreme importance to numerous technological applications [1,2]. The relationship between adhesion and surface energy between two macroscopic bodies has been studied in detail for the case of bulk materials. In the classical view of adhesion theory, the force required to separate two surfaces is linearly proportional to the work required to form two interfaces [3-7].

When a molecularly thin liquid film is applied to a solid surface, the surface energy of the film can vary significantly due to interaction of the liquid with the underlying surface [8-10]. For example, the dispersive interactions between a surface and a liquid film will result in a free energy that rapidly increases as the film thickness is decreased [11]. The thickness dependence of the dispersive surface energy $\gamma^{\mathrm{d}}(h)$ can be expressed in terms of the free energy of the bulk material $\gamma_{\text {bulk }}^{\mathrm{d}}$ as

$\gamma^{\mathrm{d}}(h)=\gamma_{\text {bulk }}^{\mathrm{d}}+\Delta \gamma^{\mathrm{d}}(h)$,

where $\Delta \gamma^{\mathrm{d}}(h)$ is termed the excess surface energy, which scales inversely as the square of the film thickness [3,11].

In addition to the van der Waals interactions, molecularspecific interactions, i.e., hydrogen bonding, dipole-dipole interaction, or covalent bonding, can develop between the applied liquid film and the solid substrate. The presence of these adhesive interactions, which are absent in the bulk liquid, will be manifested as a change in the polar surface energy of a molecularly thin film. In the case of hydroxylterminated perfluoropolyether (PFPE) films, the adhesive interaction strength, and hence the polar surface energy, is sensitive to the relative level of bonding between the polar end-groups of the PFPE lubricant and the polar sites on the carbon surface. The number of lubricant end-groups that 
are bonded to the carbon surface can be increased by exposure to elevated temperature. As the bonding ratio is increased, the polar surface energy component decreases. It is therefore possible in these systems to vary both the dispersive and polar surface energy components by altering the film thickness and/or bonding ratio. Thus, these films allow a quantitative investigation of the relationship between adhesion and the total surface energy. Furthermore, the ability to independently manipulate surface energy components as well as the excess surface energy yields additional information concerning the relationship between the adhesive force and the relative magnitude of dispersive and polar surface energy. Specifically, this work is intended to determine the dependence of adhesion on the total surface energy $\gamma^{\mathrm{T}}$, the individual surface energy components $\gamma^{\mathrm{d}}$ and $\gamma^{\mathrm{p}}$, and the excess surface energy components $\Delta \gamma^{\mathrm{d}}, \Delta \gamma^{\mathrm{p}}$.

\section{Theoretical Background}

Adhesion between two macroscopic bodies for the case of bulk materials has been thoroughly investigated [3]. Derjaguin's approximation [12] allows one to express the adhesive force $F_{\text {adh }}$ between two surfaces as the product of a geometric factor $g$ and the work of adhesion $W_{\text {adh }}$ between flat plates of infinite lateral dimensions as $[3,12]$ :

$F_{\text {adh }}=g W_{\text {adh }}$.

For contact between two curved surfaces, the geometric factor $g$ is given by $g=2 \pi\left(\frac{R_{1} R_{2}}{R_{1}+R_{2}}\right)$ where $R_{1}$ and $R_{2}$ are the radii of curvature of the two surfaces. For the case of a sphere of radius $R$ contacting a flat surface, the geometric factor reduces to $g=2 \pi R$.

The work of adhesion is defined by the Dupré equation [3]

$W_{\mathrm{adh}}=\gamma_{1}+\gamma_{2}-\gamma_{12}$

where $\gamma_{1}$ and $\gamma_{2}$ are the free energies of the separated surfaces, and $\gamma_{12}$ is the interfacial free energy. For bulk materials, the interfacial energy can be approximated as [3]

$\gamma_{12}=\gamma_{1}+\gamma_{2}-2\left(\gamma_{1} \gamma_{2}\right)^{1 / 2}$

When the two surfaces are identical $\left(\gamma_{1}=\gamma_{2}\right)$, the interfacial energy term vanishes, i.e., $\gamma_{12}=0$, and the work of adhesion is given by $W_{\mathrm{adh}}=2 \gamma$. This leads to the wellknown Bradley equation [4] for the adhesive force $F_{\text {adh }}$ between two identical surfaces,

$F_{\text {adh }}=4 \pi R \gamma$.

This functional form of the adhesive force, which is also known as the Derjaguin, Muller, and Toporov (DMT) model [5], is applicable to the adhesion between two rigid surfaces with high surface energy. When the contacting surfaces are elastic, the adhesive force is better approximated by the Johnson, Kendall, and Roberts (JKR) model [6]

$\mathrm{F}_{\mathrm{adh}}=3 \pi \mathrm{R} \gamma$.

It is immediately apparent from comparison of Eqs. 5 and 6 , that both equations predict a linear dependence of the adhesive force on the total surface energy. The difference between these two models is simply the form of the geometric factor, i.e., $g=4 \pi R$ for the DMT model and $g=3 \pi R$ for the JKR model.

In the case of two solid surfaces separated by a bulk liquid, the adhesive force increases relative to either Eqs. 5 or 6 as a result of meniscus formation [13]. The Laplace pressure generated in the liquid meniscus requires additional work to separate the surfaces. The Laplace pressure contribution to the adhesive force is given by [3]

$F=g \gamma \cos \theta$

where $\theta$ is the angle made between the liquid film and the solid surfaces. Therefore, the total adhesive force required to separate two solid surfaces in the presence of a meniscus can be written as

$F=g \gamma(1+\cos \theta)$.

In the present work, we measure the surface energy $\gamma$ of a carbon surface coated with a molecularly thin PFPE film using contact angle goniometry. Subsequently, we determine the adhesive force $F_{\text {adh }}$ between an AFM tip and the same PFPE-coated surfaces (see Fig. 1a). We then compare the experimental results with expectations based on the theoretical equations presented above.

\section{Experimental}

\subsection{Materials and Chemical Composition}

The measurements were conducted on PFPE-lubricated magnetic recording disks comprised of an AlMg substrate, a cobalt-based magnetic film, and a $3 \mathrm{~nm}$ nitrogenated carbon overcoat $\left(\mathrm{CN}_{\mathrm{X}}\right)$. The RMS roughness of these surfaces was measured to be $0.27 \pm 0.03 \mathrm{~nm}$. The carbon surfaces were coated with hydroxyl-terminated perfluoropolyether (PFPE-OH), commercially known as Fomblin Zdol. This material is a random copolymer of perfluoromethylene oxide and perfluoroethylene oxide repeat units terminated on both ends by hydroxyl moieties,

$\mathrm{HO}-\mathrm{CH}_{2}-\mathrm{CF}_{2}-\mathrm{O}-\left(\mathrm{CF}_{2} \mathrm{CF}_{2} \mathrm{O}\right)_{p}-\left(\mathrm{CF}_{2} \mathrm{O}\right)_{q}-\mathrm{CF}_{2}-\mathrm{CH}_{2}-\mathrm{OH}$.

The PFPE-OH used in this study was fractionated. Nuclear magnetic resonance spectroscopy was used to characterize the average molecular weight for the PFPE$\mathrm{OH}$ as $2540 \mathrm{amu}$. The polydispersity was found to be 1.03 
Fig. 1 a Schematic of the contact of an AFM tip on a flat, carbon-coated surface separated by a molecularly thin lubricant film. b Typical data from a force-distance measurement (a)

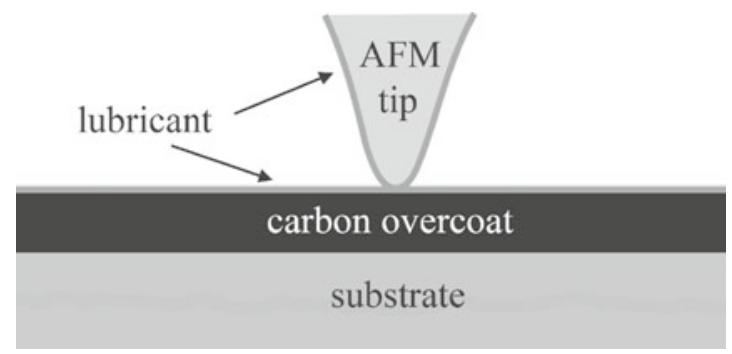

(b)

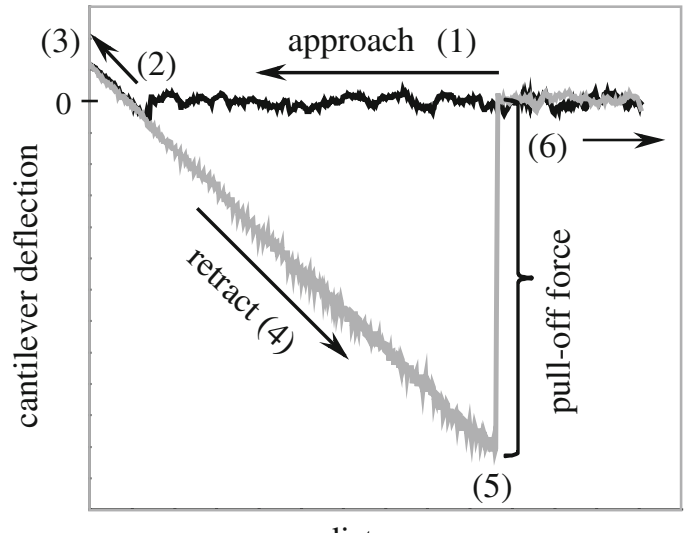

(1)

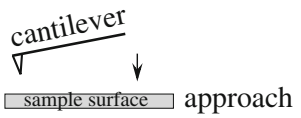

(2)

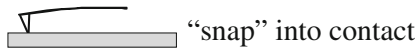

(3)

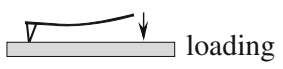

(4)

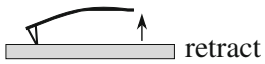

(5)

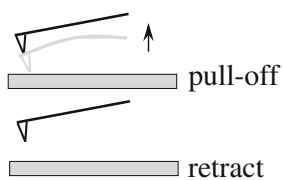

as determined by gel permeation chromatography (GPC) by the supplier. Throughout the article the lubricant is referred to as Zdol 2540. Films were applied to the amorphous carbon surface via dip-coating in dilute solutions of the PFPE-OH dissolved in perfluorohexane solvent [14].

Two different sets of samples were produced. In the first set, the PFPE-OH film thickness applied to the carbon surface was varied between 0.16 and $2.31 \mathrm{~nm}$. The thickness was controlled by varying the PFPE-OH concentration in the perfluorohexane solution, the pull-rate, or both. The applied film thickness was determined using Fourier transform infrared spectroscopy (FTIR). The thickness values obtained from FTIR spectroscopy were calibrated with measurements made using X-ray reflectivity [15]. The film thickness of all samples studied was below the "critical de-wetting" thickness [16, 17], which was determined to be $2.0 \pm 0.1 \mathrm{~nm}$ for the materials used.

A second set of samples was prepared in which the bonding ratio of the total PFPE-OH film was varied between 15 and $93 \%$ at a constant film thickness of $1 \pm 0.05 \mathrm{~nm}$. These samples were prepared in the following way. After dip-coating of the disks, bonding of the PFPE-OH films on the disk surface was increased via annealing at elevated temperatures (typically $90{ }^{\circ} \mathrm{C}$ ) for different time periods. The bonded fraction was determined by measurement of the total applied film thickness and the film thickness remaining after solvent extraction of the mobile PFPE-OH component [15]. The bonding ratio used herein is defined as the thickness of the bonded PFPE-OH film divided by the target film thickness [11].

\subsection{Surface Energy and AFM Adhesion Measurements}

The surface energy of the PFPE-OH-coated carbon surfaces was determined using contact angle goniometry $[3,8$, 10] (VCA Optima-AST Products, Inc.). The dispersive and polar surface energy components of the PFPE films, $\gamma_{s}^{\mathrm{d}}$ and $\gamma_{\mathrm{s}}^{\mathrm{p}}$, were obtained from contact angle measurements performed using hexadecane $\left(\gamma_{\mathrm{L}}^{\mathrm{d}}=27.5 \mathrm{~mJ} / \mathrm{m}^{2}\right)$ and water $\left(\gamma_{\mathrm{L}}^{\mathrm{d}}=21.8 \mathrm{~mJ} / \mathrm{m}^{2}\right.$, and $\left.\gamma_{\mathrm{L}}^{\mathrm{p}}=51.0 \mathrm{~mJ} / \mathrm{m}^{2}\right)$ as reference liquids $[9,11]$. The contact angle was determined and the results were averaged over four measurements.

Force-distance measurements with an atomic force microscope (Nanosurf, Mobile S) were used to measure the adhesive force between the PFPE-lubricated carbon surface and the AFM tip ( $\mathrm{Si}_{3} \mathrm{~N}_{4}$-tip). A typical force-distance measurement is shown in Fig. $1 \mathrm{~b}$ for a complete approach and retract cycle of the AFM tip. The measurement is done in the following sequence. First, the AFM tip is brought into close proximity to the surface (line 1 ) by lowering the cantilever towards the sample surface. At point (2), the AFM tip "snaps" into contact with the sample surface due 
to attractive van der Waals forces, deflecting the cantilever downward. Further approach results in an upward cantilever deflection and a small normal force applied to the sample surface (3). Subsequently, the cantilever is retracted from the sample surface (4). The AFM tip stays initially in contact with the sample surface due to adhesion forces, bending the cantilever downward. The AFM tip separates when the force applied to the cantilever exceeds the adhesion force between the tip and the surface (point 5). At point (6) the AFM is fully detached from the surface and the cantilever shows zero deflection. The magnitude of the cantilever deflection at point (5) is proportional to the adhesive force. The adhesion force values were determined by averaging the results of four force-distance measurements and multiplying the cantilever deflection by the cantilever spring constant of $0.2 \mathrm{~N} / \mathrm{m}$ (provided by the manufacturer). We note that the cantilever spring constant can have an inaccuracy of up to $30 \%$ from the value provided by the manufacturers.

Prior to all adhesion measurements, the AFM tip was brought into contact with the surface for a period of approximately $15 \mathrm{~min}$ to allow the tip to come into equilibrium with the PFPE-OH-coated surface.

\section{Results}

The results of the surface energy measurements conducted on the PFPE-OH films of varying thickness are summarized in Fig. 2a and b. As shown in Fig. 2a, the dispersive surface energy component of the PFPE-OH-coated surface $\gamma_{\mathrm{S}}^{\mathrm{d}}$ depends strongly on the PFPE-OH film thickness. In particular, we observe that the dispersive surface energy decreases monotonically with increasing film thickness, asymptotically approaching the value of the bulk PFPE-OH liquid $\gamma_{\text {bulk }}^{\mathrm{d}}=15.6 \mathrm{~mJ} / \mathrm{m}^{2}$ for the thickest films studied. The functional dependence of the dispersive surface energy on film thickness $\gamma^{\mathrm{d}}(h)$ can be expressed as [11],

$\gamma^{\mathrm{d}}(h)=\gamma_{\text {bulk }}^{\mathrm{d}}+\Delta \gamma^{\mathrm{d}}(h)$.

where $\Delta \gamma^{\mathrm{d}}(h)$ represents the surface energy that is in excess of the bulk value $\gamma_{\text {bulk }}^{\mathrm{d}}$ for a film of thickness $h$. It is apparent that the minimum surface energy is equal to that of the bulk value of Zdol [16]. The thickness dependence of this quantity, which we term the excess surface energy $\Delta \gamma^{\mathrm{d}}(h)$, can be described using $[10,18]$

$2 \Delta \gamma^{\mathrm{d}}(h)=\frac{A *}{12 \pi(h+d)^{2}}$

where $A^{*}$ is the effective Hamaker constant and $d$ is a constant. The fit to the data presented in Fig. $2 \mathrm{a}$ was obtained using $\gamma_{\text {bulk }}^{\mathrm{d}}=15.6 \mathrm{~mJ} / \mathrm{m}^{2}, A^{*}=0.96 \times 10^{-19} \mathrm{~J}$ and $d=0.17 \mathrm{~nm}$.
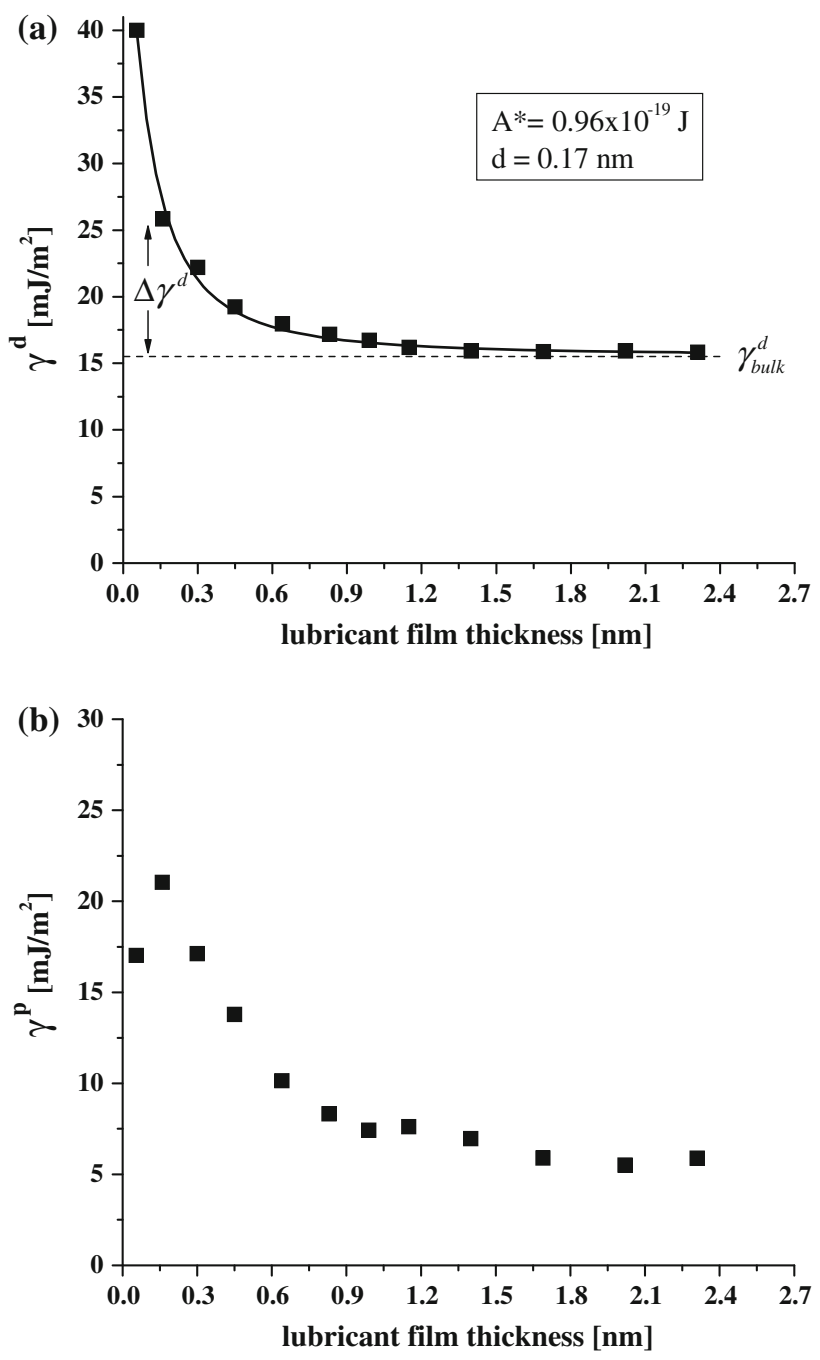

Fig. 2 a Dispersive surface energy component, $\gamma^{\mathrm{d}}$, for Zdol 2540 on a carbon-coated surface as a function of Zdol film thickness. Solid line fit to the data is based on Eq. 9 (in combination with Eq. 10) using an effective Hamaker constant of $A *=0.96 \times 10^{-19} \mathrm{~J}$. Dashed line at $15.6 \mathrm{mN} / \mathrm{m}^{-2}$ denotes the bulk value of the dispersive surface energy, $\gamma_{\text {bulk }}^{\mathrm{d}}$. b The dependence of the Zdol 2540 polar surface energy, $\gamma^{\mathrm{p}}$, on applied film thickness

The dependence of the polar surface energy component $\gamma^{\mathrm{p}}$ on PFPE-OH film thickness is shown in Fig. 2b. We observe that the polar surface energy also decreases with increasing film thickness, approaching a minimum of $5.5 \mathrm{~mJ} / \mathrm{m}^{2}$ for the thickest films studied. This dependence is very similar to previous results by $[8,9]$ on PFPE-OH films in which a minimum in the value of the surface energy was observed at a thickness corresponding to one monolayer.

The dependence of the surface energy components $\gamma^{\mathrm{d}}$ and $\gamma^{\mathrm{p}}$ on the bonding ratio of PFPE-OH is presented in Fig. 3a and b. We observe from Fig. 3a that the dispersive surface energy component $\gamma^{\mathrm{d}}$ is nearly independent of the bonding ratio. However, the polar component of the 

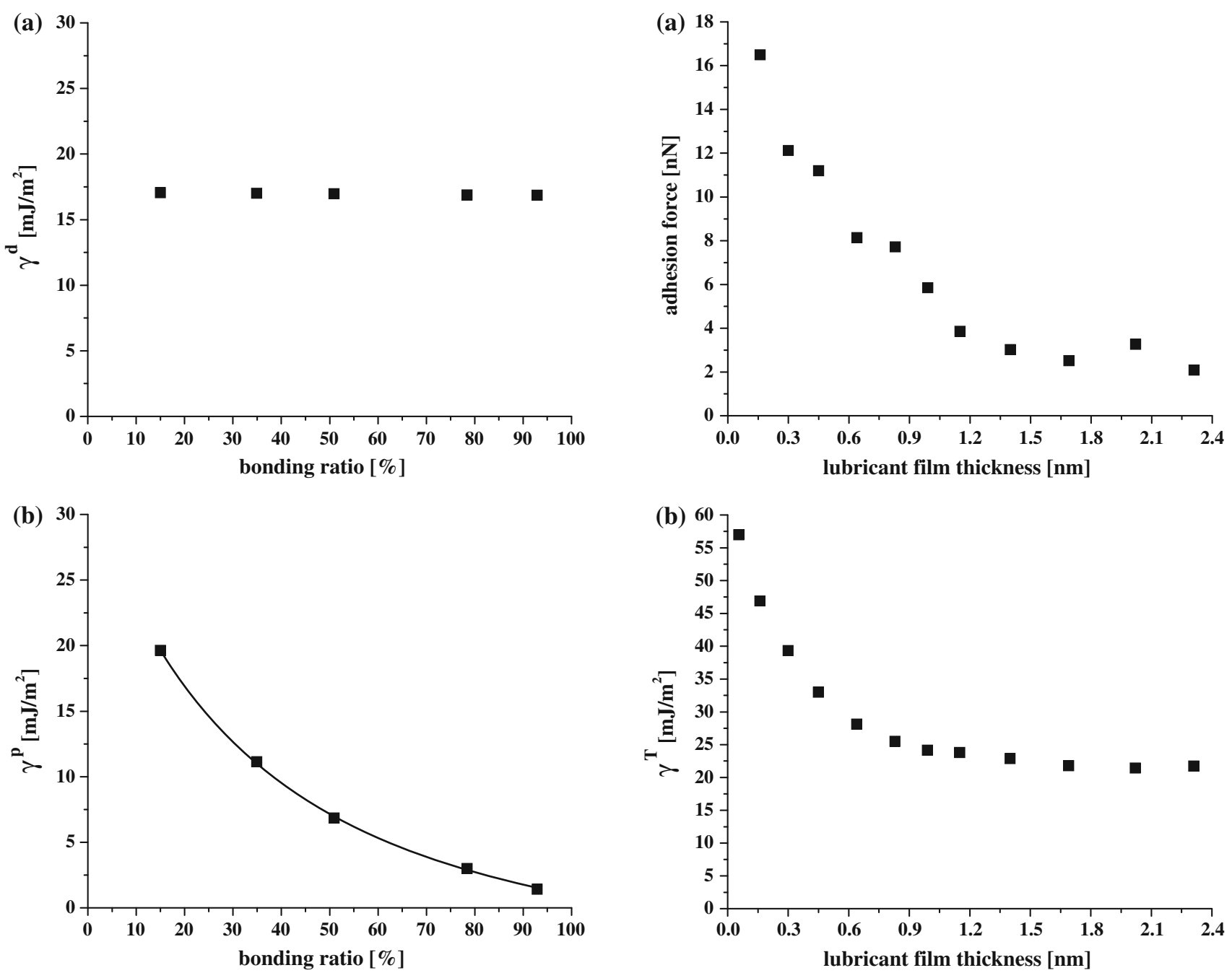

Fig. 3 The dispersive (a) and polar (b) surface energy components as a function of bonding ratio for $1 \mathrm{~nm}$ Zdol 2540 films

surface energy $\gamma^{\mathrm{p}}$ (Fig. 3b) decreases with increasing bonding ratio and approaches $\gamma_{\min }^{\mathrm{p}}=0.5 \pm 0.5 \mathrm{~mJ} / \mathrm{m}^{2}$ at $100 \%$ bonding of the film. The drop in $\gamma^{\mathrm{p}}$ with increasing bonding ratio has been interpreted by Waltman et al. [8] in terms of attractive hydrogen bonding between the hydroxyl end-groups and the polar sites on the carbon surface. In particular, the authors suggested that an increase in the bonding ratio results in a reduction of the number of "free", or non-interacting, hydroxyl end-groups of the PFPE lubricants as well as the number of polar surface sites. This neutralization of polar sites on both the lubricant and the carbon surface appears to be responsible for the experimentally observed reduction in polar surface energy component.

In analogy to Eq. 9, we can express the total polar surface energy as:

$\gamma^{\mathrm{p}}(h)=\gamma_{\min }^{\mathrm{p}}+\Delta \gamma^{\mathrm{p}}$

Fig. 4 a Adhesion force as a function of the applied PFPE-OH film thickness compared to $\mathbf{b}$ total surface energy $\gamma^{T}$ (sum of dispersive and polar component) as a function of the applied PFPE-OH film thickness

where $\gamma_{\min }^{\mathrm{p}}$ corresponds to the polarity of the carbon-PFPE system at complete saturation of the polar sites (100\% bonding of the film). Based on our experimental measurements, this results in a minimum polar surface energy of $\gamma_{\min }^{\mathrm{p}}=0.5 \pm 0.5 \mathrm{~mJ} / \mathrm{m}^{2}$, i.e., $\gamma_{\min }^{\mathrm{p}}$ is negligible in comparison to $\Delta \gamma^{\mathrm{p}}$ and thus, Eq. 11 becomes $\gamma^{\mathrm{p}}=\Delta \gamma^{\mathrm{p}}$.

Force-distance measurements were conducted on both sets of PFPE-coated surfaces. The magnitude of the cantilever deflection at the point of separation from the surface was found to be strongly dependent on both the PFPE-OH film thickness and the bonding ratio. The dependence of adhesion on film thickness is shown in Fig. 4a, and the dependence of adhesion on the bonding ratio is shown in Fig. 5a. As is apparent from these figures the adhesion between the AFM tip and the coated surface decreases with both increasing PFPE-OH film thickness and increasing 

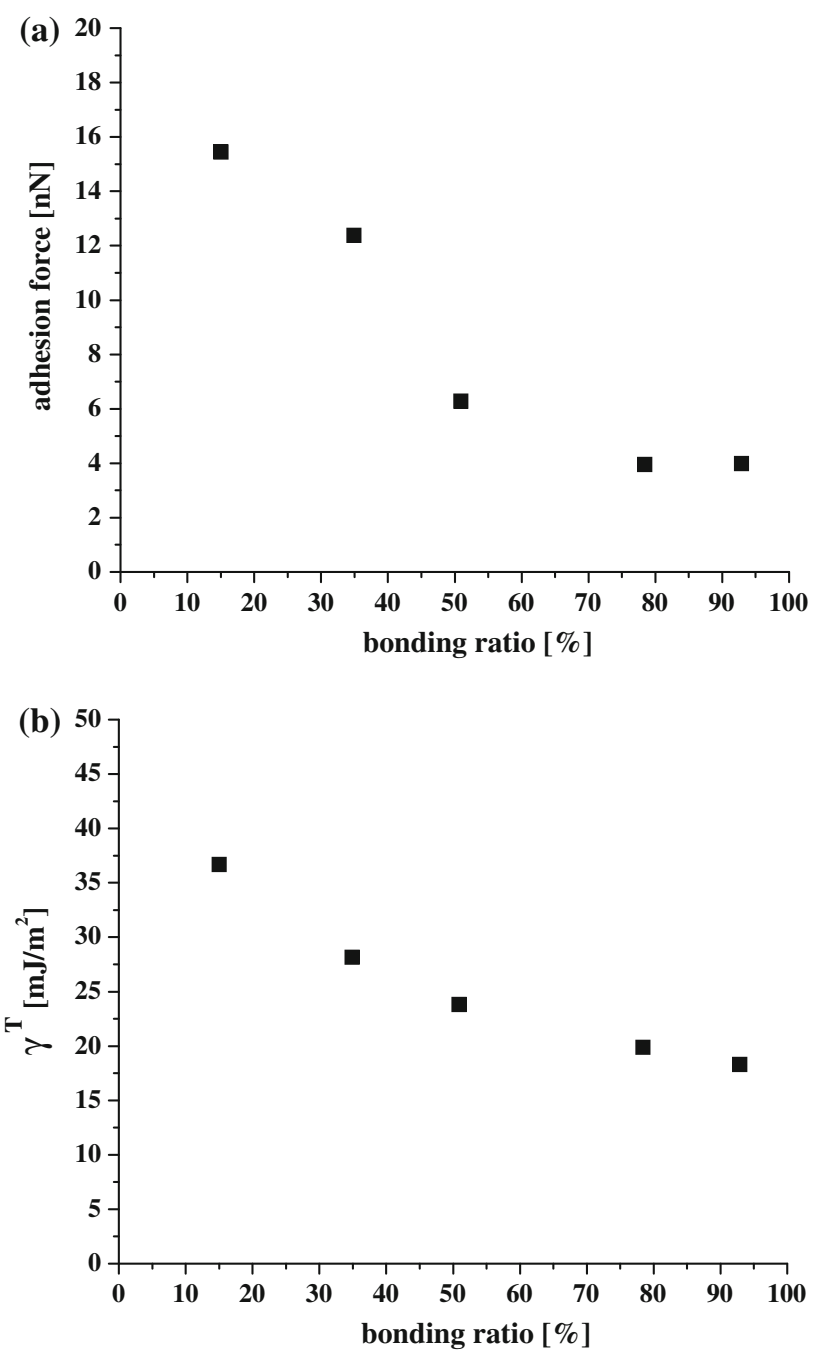

Fig. 5 a Adhesion force as a function of the PFPE-OH bonding ratio compared to $\mathbf{b}$ total surface energy $\gamma^{\mathrm{T}}$ (sum of dispersive and polar component) as a function of the PFPE-OH bonding ratio

bonding ratio. The adhesion results are compared with the corresponding total surface energies (the sum of the polar and dispersive surface energy component) in Fig. $4 \mathrm{~b}$ for the film thickness and in Fig. $5 \mathrm{~b}$ for the bonding ratio. The results indicate a strong correlation between the surface energy of the PFPE-OH-coated surfaces and the adhesion measurements.

\section{Discussion}

The dependence of adhesion force on total film surface energy is shown in Fig. 6 for both sets of PFPE films (lubricant thickness and bonding ratio) studied. We observe a nearly linear dependence between adhesion force and total surface energy. We note that the slope of the line for the PFPE-OH bonding ratio data is steeper than that for the

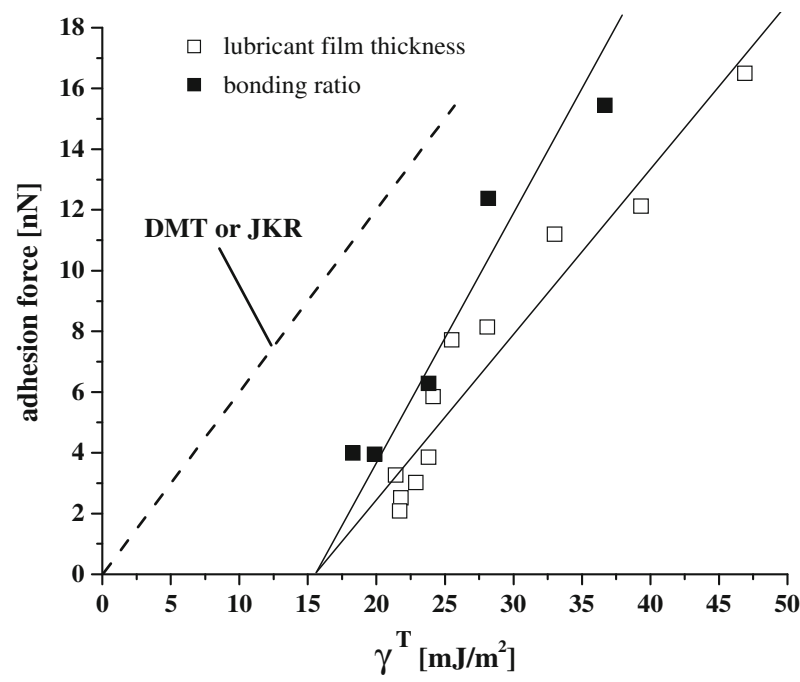

Fig. 6 Adhesion force as a function of total surface energy $\gamma^{\mathrm{T}}$ for varying film thickness (open square), and varying bonding ratios (filled square) of Zdol 2540 films including linear fits to the data (-). The dashed line illustrates an example of the DMT or JKR model

lubricant thickness data. We believe that this result is related to the difference in the radii or cantilever stiffness of the AFM tips that were used for the two experiments. In particular, the AFM tip radius or cantilever stiffness of the AFM probe that was used to collect the adhesion data of the PFPE-OH bonding ratio samples was slightly larger than that used for the samples with different lubricant film thickness. However, the change in the spring constant or the tip radius only influences the geometric factor $g$ and will not influence our experimental findings as shown below.

The results of these measurements show that there is no evidence for meniscus formation at the contact (see Fig. 4). This is consistent with previous results on molecularly thin PFPE films from the literature [19-21].

While the observed linear relationship between surface energy and adhesion qualitatively agrees with that predicted by adhesion theory, the results of the present work illustrate that adhesion between surfaces separated by molecularly thin PFPE films vanishes at a finite value of surface energy (Fig. 6). This observation fundamentally differs from the predictions obtained using the theoretical results from the DMT model or the JKR model. In particular, both the DMT and JKR models predict that adhesion vanishes when the surface energy goes to zero. This is shown schematically by the dashed line in Fig. 6.

The experimental results shown in Fig. 6 indicate that the adhesion between surfaces separated by molecularly thin films does not scale with the total surface energy $\gamma^{\mathrm{T}}$. Extrapolation of the experimental data obtained in this work indicates that the adhesive force between the AFM tip 


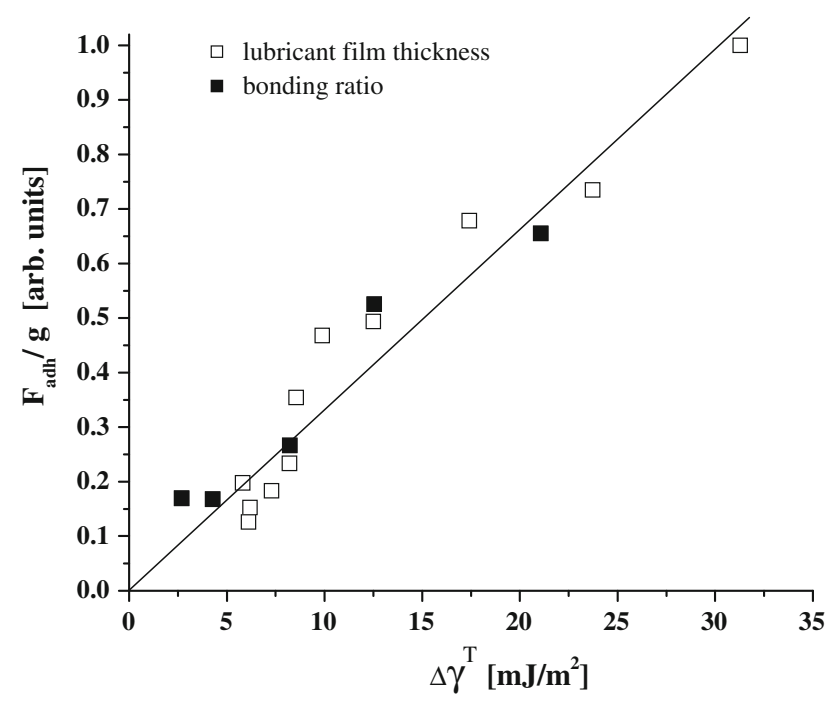

Fig. 7 The quantity $F_{a d h} / g$ was normalized arbitrarily to 1 for the highest adhesion measured as a function of the sum of the excess surface energy $\Delta \gamma^{T}$ (the sum of the dispersive and polar component, $\left.\Delta \gamma^{d}+\Delta \gamma^{p}\right)$

and the PFPE-OH-coated surface vanishes at approximately $15-16 \mathrm{~mJ} / \mathrm{m}^{2}$. Since this surface energy value is close to $\gamma_{\text {bulk }}^{\mathrm{T}}=\gamma_{\text {bulk }}^{\mathrm{d}}+\gamma_{\text {min }}^{\mathrm{p}}$, our data suggests that the adhesive force between two contacting surfaces separated by molecularly thin films is related to the total excess surface energy $\Delta \gamma^{\mathrm{T}}=\Delta \gamma^{\mathrm{d}}+\Delta \gamma^{\mathrm{p}}$ rather than the total surface energy $\gamma^{\mathrm{T}}$.

In order to demonstrate this relationship, we plot the adhesive force versus the excess surface energy $\Delta \gamma^{\mathrm{T}}$ as shown in Fig. 7. We note that the quantity $F_{\text {adh }} / g$ in Fig. 7 was normalized to 1 for the highest adhesion measured. Therefore, any errors that may have been caused by the cantilever spring constant or a change in the AFM tip radius are eliminated. Figure 7 clearly shows that the adhesion scales linearly with $\Delta \gamma^{\mathrm{T}}$ and vanishes as $\Delta \gamma^{\mathrm{T}} \rightarrow 0$. We therefore conclude that the adhesion between two surfaces separated by a molecularly thin PFPE-OH film is described by the following relationship:

$F_{\text {adh }}=g \Delta \gamma^{\mathrm{T}}$.

where $\Delta \gamma^{\mathrm{T}}$ is the total excess surface energy of the molecularly thin PFPE lubricant.

\section{Conclusion}

The experimental results suggest that a modification of the classical adhesion theory may be necessary to describe the adhesion between surfaces separated by molecularly thin liquid films. Specifically, the article shows that adhesion in these systems is dictated by the excess surface energy, $\Delta \gamma$, and not the total surface energy, $\gamma^{\mathrm{T}}$.
Open Access This article is distributed under the terms of the Creative Commons Attribution Noncommercial License which permits any noncommercial use, distribution, and reproduction in any medium, provided the original author(s) and source are credited.

\section{References}

1. Bhushan, B.: Nanotribology and Nanomechanics-An Introduction. Springer-Heidelberg, Germany (2005)

2. Shinji, Y., Israelachvili, J.: Friction and adhesion hysteresis of fluorocarbon surfactant monolayer-coated surfaces measured with the surface forces apparatus. J. Phys. Chem. B 102, 234-244 (1998)

3. Israelachvili, J.N.: Intermolecular and Surface Forces, 2nd edn. Academic Press, London (1992)

4. Bradley, R.S.: The cohesive force between solid surfaces and the surface energy of solids. Philos. Mag. 13, 853 (1932)

5. Derjaguin, B.V., Muller, V.M., Toporov, Y.P.: Effect of contact deformations on adhesion of particles. J. Colloid Interface Sci. 53, 314 (1975)

6. Johnson, K.L., Kendall, K., Roberts, A.D.: Surface energy and contact of elastic solids. Proc. R. Soc. London, Ser. A 324, 301 (1971)

7. Maugis, D.: Adhesion of spheres: the JKR-DMT transition using a Dugdale model. J. Colloid Interface Sci. 150, 243-269 (1992)

8. Waltman, R.J., Pocker, D.J., Tyndall, G.W.: Studies on the interactions between ZDOL perfluoropolyether lubricant and the carbon overcoat of rigid magnetic media. Tribol. Lett. 4, 267-275 (1998)

9. Tyndall, G.W., Leezenberg, P.B., Waltman, R.J., Castenada, J.: Interfacial interactions of perfluoropolyether lubricants with magnetic recording media. Tribol. Lett. 4, 103-108 (1998)

10. Waltman, R.J.: The interactions between Z-tetraol perfluoropolyether lubricant and amorphous nitrogenated- and hydrogenated-carbon surfaces and silicon nitride. J. Fluorine Chem. 125, 391-400 (2004)

11. Waltman, R.J., Yen, B.K., White, R.L.: The adhesion of monomolecular hydroxyl-terminated perfluoropolyether liquid films on the sputtered silicon nitride surface as a function of end group acidity and mobility. Tribol. Lett. 20(1), 69-81 (2005)

12. Derjaguin, B.V.: Untersuchungen über die Reibung und Adhäsion, IV: Theorie des Anhaftens kleiner Teilchen. Kolloid Z. 69, 155-164 (1934)

13. Bowden, F.P., Tabor, D.: The friction and lubrication of solids. Oxford University Press, London (1950)

14. Gao, C., Lee, Y.C., Chao, J., Russak, M.: Dip-coating of ultrathin liquid lubricant and its control for thin-film magnetic hard disks. IEEE Trans. Magn. 31(6), 2982-2984 (1995)

15. Toney, M.F., Mate, C.M., Pocker, K.J.: Calibrating ESCA and ellipsometry measurements of perfluoropolyether lubricant thickness. IEEE Trans. Magn. 34(4), 1774-1776 (1998)

16. Waltman, R.J., Khurshudov, A., Tyndall, G.W.: Autophobic dewetting of perfluoropolyether films on amorphous-nitrogenated carbon surfaces. Tribol. Lett. 12(3), 163-169 (2002)

17. Waltman, R.J.: The stability of ultra-thin perfluoropolyether mixture films on the amorphous nitrogenated carbon surface. J. Colloid Interface Sci. 313, 608-611 (2007)

18. Waltman, R.J., Tyndall, G.W., Pacansky, J.: Computer-modeling study of the interactions of Zdol with amorphous carbon surfaces. Langmuir 15, 6470-6483 (1999)

19. Ruths, M., Granick, S.: Rate-dependent adhesion between opposed perfluoropoly(alkyl ether) layers: dependence on chainend functionality and chain length. J. Phys. Chem. B 102, 60566063 (1998) 
20. Stanley, H.M., Etsion, I., Bogy, D.B.: Adhesion of contacting rough surfaces in the presence of sub-boundary lubrication. ASME J. Tribol. 112, 98-104 (1990)
21. Waltman, R.J., Tyndall, G.W.: Lubricant and overcoat systems for rigid magnetic recording media. J. Magn. Soc. Jpn. 26(3), 97108 (2002) 